\title{
Dark Matter searches via direct detection
}

\section{María Martínez*}

Laboratorio de Física Nuclear y Astropartículas, Universidad de Zaragoza, C/ Pedro Cerbuna 12, 50009 Zaragoza, Spain

Fundación ARAID, Av. de Ranillas 1D, 50018 Zaragoza, Spain

E-mail: mariameunizar.es

The evidence for dark matter is overwhelming from cosmological observations at small and large scales. Yet, its existence is inferred indirectly, through its gravitational effects, but its nature is still unknown. Among the preferred dark matter particle candidates are new particles beyond the standard model with weak scale interaction with the known particles and mass in the $\mathrm{GeV}$ to hundreds of $\mathrm{GeV}$ scale. They are generically called Weakly Interacting Massive Particles (WIMPs). Thanks to their (weak but not zero) coupling with ordinary matter, they can be detected with a particle detector in the Earth. I will review the concepts of direct detection of WIMPs and the main experimental efforts to search for them, including the only positive result up to date (DAMA/LIBRA) and the ongoing experiments attempting to confirm or refute this signal in a model independent way. I will also discuss future experiments, and their ability to explore new regions of the WIMPs parameter space or go beyond the current ultimate limits.

Corfu Summer Institute 2018 "School and Workshops on Elementary Particle Physics and Gravity" (CORFU2018)

31 August - 28 September, 2018

Corfu, Greece

${ }^{*}$ Speaker. 


\section{Introduction}

More than $95 \%$ of our Universe is not explained in the frame of the standard model of the particle physics. Ordinary matter represents only $5 \%$ of the energy content of the Universe, while around $27 \%$ is constituted by a new type of particle(s) and which we generically refer to as Dark Matter (DM). The remaining 70\%, of unknown nature, is responsible for the accelerated expansion of the Universe and we call it Dark Energy. The evidence for DM is overwhelming at the astrophysical and cosmological scale since both our galaxy and the observable Universe would be inexplicable without the additional gravity that it produces. However, there is still no data on its nature [1].

Baryonic DM can be excluded not only by the precision measurements of the cosmic microwave background (CMB) power spectrum [2], which constrains the baryonic Universe content to $\sim 5 \%$, but also by the agreement between big bang nucleosynthesis predictions and measurements of the primordial abundance of light elements [3]. Primordial black holes (PBHs) produced before the big bang nucleosynthesis in the very early Universe have been recently re-examined as DM candidates, as they do not enter in the $5 \%$ baryonic component. But a number of Galactic and cosmological observations disfavor PBHs as the main DM component (see for example [4]). Neutrinos decoupled at a temperature $\mathrm{k}_{B} \mathrm{~T} \sim 2-3 \mathrm{MeV}$, when the reaction $e^{+}+e^{-} \rightleftharpoons v+\bar{v}$ was frozen, leaving behind a density of fossil neutrinos. They are part of what we call hot dark matter (HDM), that is, matter that was relativistic at the time when the structures were formed on astrophysical scale and which cannot explain the current observations.

In order to solve the DM puzzle we need to find a cold dark matter (CDM) solution beyond the standard model of particle physics. The candidates that have been proposed so far cover many orders of magnitude in mass and interaction cross section. Among them, those that have raised more interest are the generically called weakly interacting massive particles (WIMPs). They are appealing thanks to the remarkable coincidence of two facts: if they existed in the early Universe, their relic abundance now is in agreement with the DM observed one, and they are predicted independently to the DM problem by extensions of the standard model. A popular candidate is the lightest supersymmetric particle (LSP) in supersymmetric extensions, e.g. the neutralino (the supersymmetric partner of the gauge and Higgs bosons), the gravitino (superpartner of the graviton), or the sneutrino (superpartner of the neutrino) [5]. The supersymmetric models usually predict WIMPs with masses larger than $30 \mathrm{GeV}$. Another appealing possibility is the lightest Kaluza-Klein particle in theories with universal extra dimensions [6].

An alternative to the WIMP paradigm are axions, firstly proposed by Peccei and Quinn as a solution to the strong charge-parity problem in quantum chromodynamics [7]. More generally, axion-like particles (ALPs), although very light, would constitute CDM because they were produced non-thermally. There are a large number of efforts devoted to their detection (see for example [8]), but this kind of searches are beyond the scope of this paper.

In recent years, a plethora of new DM candidates has emerged in the field, together with innovative ideas to detect them. Many of these models rely on the hidden-sector DM, i.e., DM that interacts through a new force. The parameter space of these candidates extends well below the $\mathrm{GeV}$ scale. New experiments with modest investment but exploiting original ultra-low energythreshold techniques are emerging to explore these well-motivated scenarios [9]. In the following I 
will focus on the standard WIMP direct detection analysis, however I will also review some of the most interesting proposals that are being developed for low-mass DM.

\section{Dark Matter direct detection}

WIMPs can be detected by elastic scattering off nuclei, as they interact weakly with standard model particles. The local DM density is $\rho_{W} \sim 0.4 \mathrm{GeV} / \mathrm{cm}^{2}$ [10] with a RMS velocity of about $270 \mathrm{~km} / \mathrm{s}$. This implies a flux at the Earth of $10^{8}-10^{10} \mathrm{~s}^{-1} \mathrm{~m}^{-2}$ for a WIMP mass $m_{W} \sim 100 \mathrm{GeV}$. The DM interaction rate in the detector is proportional to the number of target nuclei $N_{T}=M_{d e t} / m_{N}$, where $M_{\text {det }}$ is the detector mass and $m_{N}$ the nuclear mass number, the number density of WIMPs $n_{W}=\rho_{W} / m_{W}$, the relative velocity with respect to the detector $v$ and the interaction cross section $\sigma$, that in general depends on the WIMP energy and the transferred momentum. So, taking into account a WIMP velocity distribution in the detector reference system given by $f(v)$, we can write the differential rate as

$$
\frac{d R}{d E_{R}}=\frac{\rho_{W}}{m_{W}} \frac{M_{d e t}}{m_{N}} \int_{v_{\min }}^{v_{\max }}, v f(v) \frac{d \sigma}{d E_{R}}\left(v, E_{R}\right) d v^{3}
$$

where $E_{R}$ is the nucleus recoil energy, that depends on the scattering angle. Due to the energy threshold of the detector, not all the phase space of the WIMP velocity distribution is accessible. The minimal velocity of the WIMP in order to produce a nuclear recoil of energy $E_{R}$ is given by $v_{\min }\left(E_{R}\right)=\frac{m_{W}+m_{N}}{m_{W}} \sqrt{\frac{2 E_{R}}{2 m_{N}}}$. As regards the upper limit of the integral, it corresponds to the galaxy escape velocity $v_{\text {esc }}$, subject to large uncertainties [10].

The differential cross section WIMP-nucleus $\frac{d \sigma}{d E_{R}}$ can be written as the product of a punctual cross section and a form factor $F$ that takes into account the nucleus size and encodes the dependence on the momentum transfer. In addition, in the non-relativistic velocity limit the cross section can be decomposed into two contributions: the spin-independent (SI) and the spin-dependent (SD):

$$
\sigma F^{2}\left(E_{R}\right)=\sigma_{S I} F_{S I}^{2}\left(E_{R}\right)+\sigma_{S D} F_{S D}^{2}\left(E_{R}\right),
$$

where $\sigma_{S I}$ and $\sigma_{S D}$ are the SI and SD contributions to the WIMP-nucleon cross-section. The event rate for SI interaction is greatly enhanced due to coherence with respect to the SD one. Furthermore, for spin-zero isotopes only the SI component is present. If we assume that the SI coupling is isospin-invariant $\left(f_{p}=f_{n}\right.$, being $f_{p}$ and $f_{n}$ the corresponding effective couplings to protons and neutrons respectively) we can write:

$$
\frac{d \sigma_{S I}}{d E_{R}}\left(v, E_{R}\right) \propto A^{2} f_{p} \sigma_{S I} F_{S I}^{2}\left(E_{R}\right)
$$

where $\mathrm{A}$ is the atomic number of the nucleus. The SD part can be written as

$$
\frac{d \sigma_{S D}}{d E_{R}}\left(v, E_{R}\right) \propto \frac{J+1}{J}\left[a_{p}\left\langle S_{p}\right\rangle+a_{n}\left\langle S_{n}\right\rangle\right] \sigma_{S D} F_{S D}^{2}\left(E_{R}\right),
$$

where $J$ is the total nuclear spin, $a_{p}$ and $a_{n}$ are the effective couplings WIMP-proton and WIMPneutron for SD interaction and $\left\langle S_{p}\right\rangle$ and $\left\langle S_{n}\right\rangle$ are the expectation values of the total spin operators for protons and neutrons in the target nucleus, which have to be calculated according to a nuclear model. 
Recently, more general interpretations of DM interactions have been proposed in terms of more general effective field theories (EFT), containing, for instance, velocity suppressed operators (see for instance [11, 12]).

The DM velocity distribution is commonly described by an isotropic Maxwell-Boltzmann profile:

$$
f(\vec{v}) d^{3} \vec{v}=\frac{1}{v_{0}^{3} \pi^{3 / 2}} e^{-\frac{|\vec{v}|^{2}}{v_{0}^{2}}} d^{3} \vec{v}
$$

where $v_{0}$ is the velocity of the Sun around the galactic center. The dispersion velocity $v_{r m s}$ is related to $v_{0}$ via $v_{r m s}=\sqrt{3 / 2} v_{0}$. This model is often called the standard halo model (SHM). Besides the SHM does not provide a realistic description of the DM halo, it is by far the simplest one and consequently it is commonly adopted for comparison between direct detection experiments.

Finally, it is important to mention also the effect of the quenching factor, or relative efficiency factor (REF). It measures the relative efficiency in the conversion into a measurable signal of a nuclear recoil energy deposition with respect to that corresponding to an electron recoil. Usually $\gamma$ sources are used to calibrate the experimental spectrum. Consequently, the energy is expressed in electron-recoil equivalent (keVee). In order to obtain the corresponding nuclear recoil energy, the REF has to be measured and applied.

In order to identify dark matter interactions, not only huge detectors are needed, but there are required ultra-low background experimental conditions. The experiments have to be installed underground to avoid the cosmic rays showers. They have to be carefully designed to avoid the radioactive background (internal and external). Especially important is the use of techniques of fiducialization and particle discrimination, as the DM interaction candidates are nuclear recoils and most of the backgrounds produce electron recoils. But the ultimate background in the search for DM come from neutrinos. The coherent neutrino-nucleus scattering [13], already measured by the COHERENT collaboration [14], produce a nuclear recoil signal indistinguishable from the DM one. Solar pp and ${ }^{7} \mathrm{Be}$ neutrinos produce an (almost)irreducible background in the low-mass WIMP region, while atmospheric and diffuse supernova neutrino background (DSNB) do so for WIMP masses $>10 \mathrm{GeV}[15,16]$. New strategies are needed to go beyond the so called neutrino floor.

\section{Direct detection status}

The direct detection search, started in the nineties with conventional scintillation and germanium detectors, is now a global effort. The application of double-readout techniques at the end of the nineties marked a new era in the DM searches leading to a spectacular increase of the sensitivity. As the energy partition among the two readout channels depends on the nature of the interacting particle, these techniques provide a event-by-event discrimination between nuclear recoils and other $(\alpha, \beta, \gamma)$ backgrounds. In the following I will outline the current status of the searches both in the SI and SD sectors (see $[17,18,19]$ for recent reviews).

\subsection{Spin independent direct detection}

The current experimental upper limits for the spin independent WIMP-nucleon interactions as a function of the WIMP mass are summarized in Fig. 1. The SI direct detection efforts can be 


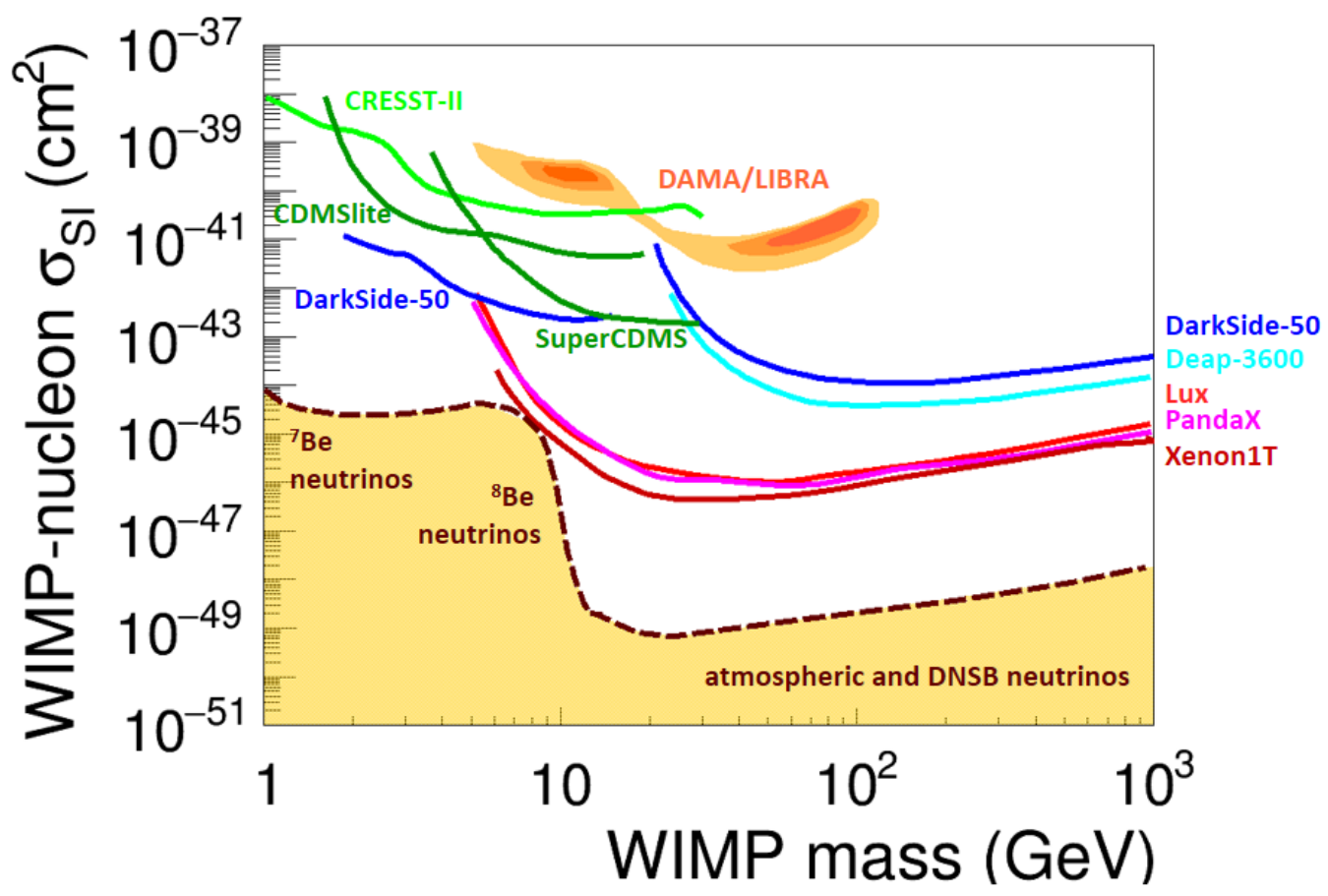

Figure 1: Current experimental upper limits for the spin independent WIMP-nucleon interactions as a function of the WIMP mass: XENON1T [20], LUX [21], PANDAX-II [22], DEAP-3600 [23], DarkSide50 [24, 25], SuperCDMS [26], CDMSlite [27] and CRESST [28]. The neutrino floor is also shown in dashed brown line. The dark orange/orange/light orange islands correspond to the $90 \%$ C.L, $3 \sigma$ and $5 \sigma$ regions of the DAMA/LIBRA positive signal, following the analysis presented in [29].

divided in three different categories:

- The only positive experimental result up to date in direct searches comes from the study of the annual-modulation of the rate measured by the DAMA/LIBRA experiment [30]. For more than 20 years this results remains unconfirmed and in strong tension with results from other experiments. The key to solve this puzzle could be close, as a number of experiments using the same target than DAMA/LIBRA are currently ongoing. The last section of this paper is devoted to present the DAMA signal and the experimental efforts trying to confirm/refute it.

- For large masses of the WIMP, the field is dominated by dual-phase (gas and liquid) noblegas TPC experiments, either making use of Xe or Ar, both of them good scintillators. These technologies combine excellent features for the DM search: (1) high radiopurity of the target; (2) thanks to the double light readout in the liquid and gas phases, event-by-event particle discrimination is feasible; (3) 3-D position reconstruction, that allow volume fiducialization; (4) easiness to scale in mass. The most constraining upper limits come from the XENON1T experiment [20] using $\mathrm{Xe}$ as target and 1 ton $\times \mathrm{yr}$ exposure. Important results have also been obtained by other two dual-phase Xe experiments: LUX [21], with $129.5 \mathrm{~kg} \times \mathrm{yr}$ and PANDAX-II [22], with $148 \mathrm{~kg} \times$ yr. On the other hand, impressive results are obtained also with single-phase detectors that do not have background discrimination capability. They 
measure only the scintillation signal produced in the noble-liquid with a spherical tank surrounded by photomultipliers in order to increase the light collection. Thanks to the huge volumes and high radiopurity they provide very competitive limits (e.g. DEAP-3600, a 3.7 ton Ar spherical vessel [23], or XMASS with 0.8 ton Xe [31]). Despite the comparatively reduced exposure $(45 \mathrm{~kg} \times \mathrm{yr})$, competitive results are obtained by the DarkSide-50 experiment [25], especially at low WIMP masses (see below). This experiment uses Ar as target, after a intense campaign for ${ }^{39} \mathrm{Ar}$ reduction. These collaborations are proposing upgrades for the future and joining efforts, so they will dominate the field in the next decade. The Xe community is projecting a union of LUZ and ZEPLIN experiments, LZ [32] that will evolve to a multi-ton WIMP detector, DARWIN [33]. The Ar community on the other hand is doing the same. The union of DarkSide-50, DEAP, miniCLEAN and ArDM experiments is now the Global Argon Dark Matter collaboration, whose main project consist in a 20 ton dual-phase Ar TPC, DarkSide-20K [34], already in construction at LNGS. The next step will be a 300 ton experiment is in design stage. These upgrades will starting reaching the neutrino floor in the next decade.

- Below $10 \mathrm{GeV}$, an ultra-low energy threshold is essential, especially for targets with large A nuclei. The bolometric detectors, like CRESST [28], SuperCDMS [26] and CDMSlite [27], are among the experiments that provides the best limits in this region. By reducing the single-bolometer mass (and in consequence the heat capacity) they are able to lower the energy threshold down to several tens of $\mathrm{eV}$, with the additional advantage that for cryogenic detectors the REF is very close to 1 (as most of the energy goes to the heat channel). However the best sensitivity to the date in this WIMP-mass region is for a noble-gas TPC experiment: DarkSide-50 [24]. The collaboration was able to reduce the energy threshold down to 50 eVee by switching off the electron-recoil discrimination. Other interesting experiments are based on TPCs (TREX) or CCDs (DAMIC, SENSEI). But there are more and more new ideas to go even beyond the $\mathrm{GeV}$ scale using new materials and techniques, like graphene [35], superfluid He, superconductors, etc.

\subsection{Spin dependent direct detection}

As exposed in section 2, neutrons and protons contribute differently to the total spin of the target. For this reason, SD interpretation of direct detection results are usually reported assuming that WIMPs couple either only to neutrons $\left(a_{p}=0\right)$ or to protons $\left(a_{n}=0\right)$. Fig. 2 shows the current best SD limits in both cases. For n-WIMP SD coupling, best limits are obtained by dual-phase Xe experiments, due to the large fraction $(\sim 50 \%)$ of natural abundance of Xe isotopes with unpaired neutrons. In the case of Ge (the target of CDMS-II and SuperCDMS), only ${ }^{73} \mathrm{Ge}$, with a natural abundance of $7.7 \%$ can contribute, while Ar has no stable isotope with unpaired spin.

Regarding p-WIMP SD coupling, there are not many DM targets with unpaired protons, although when two-body currents are included, neutrons can contribute to the proton-only coupling, resulting in some sensibility for Xe and Ge targets. Among the unpaired $\mathrm{p}$ targets we can cite some scintillators, as NaI and CsI, but scintillation experiments with only light signal don't have nuclear-recoil discrimination capability, so they results are not competitive with other experiments that can separate the nuclear recoil component. The most sensitive isotope to $\mathrm{p}$-SD coupling is 


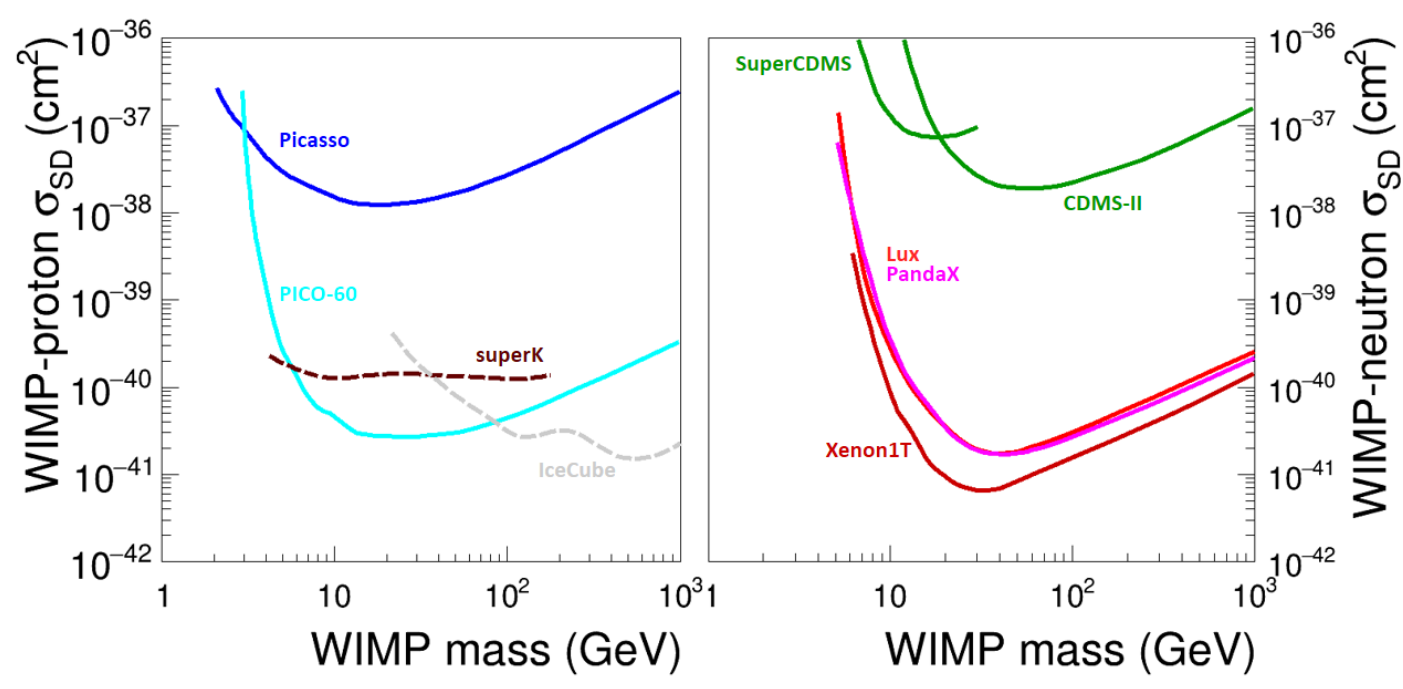

Figure 2: Current experimental upper limits for the spin dependent WIMP-nucleon interactions as a function of the WIMP mass, supposing only WIMP-proton coupling (left) or only WIMP-neutron coupling. Left panel: PICASSO [36] and PICO-60 [37]. Ice-Cube and Super-K limits are also shown. Right panel: XENON1T [20], LUX [38], PANDAX-II [22], SuperCDMS [26], CDMS-II

${ }^{19} \mathrm{~F}$, and this element is present in refrigerants like $\mathrm{C}_{3} \mathrm{~F}_{8}, \mathrm{C}_{4} \mathrm{~F}_{10}$ or $\mathrm{CF}_{3} \mathrm{I}$. These liquids, when superheated above its boiling point, can nucleate the formation of a visible bubble triggered by the deposition of a small amount of energy. The process is not triggered by low-ionizing electrons, so they detect only $\alpha$ particles (that can be rejected with high efficiency in base of other parameters) and nuclear recoils. Among the bubble chambers detectors the most relevant are COUPP [39] and PICASSO [36], which have joined in the PICO project. PICO-60, using $\mathrm{C}_{3} \mathrm{~F}_{8}$, has reported the best p-WIMP SD limits up to date [37].

\section{Beyond the neutrino floor}

The neutrino floor represents the ultimate background in DM direct detection, as solar, atmospheric and DSNB produce nuclear recoils with similar rates and energy spectra and the experiments cannot be shielded against. However supersymmetric models propose candidates below this limit. One possibility to beat the neutrino floor is to discriminate the DM from the neutrino signal by spectral analysis, but in order to do so not only huge exposures are needed, but also an improvement in the theoretical estimation and experimental determination of the neutrino fluxes. Current efforts are focus on exploiting distinctive signals, as could be the following:

- Combining data from experiments using different targets can improve the dark matter discovery potential due to target complementarity [40], as the neutrino floor is different is shape and level for the different targets.

- An annual modulation is expected in the DM rate induced by the movement of the Earth around the Sun. This signature could allow to distinguish the DM signal from the neutrino one. I will devote next section to study this effect. 
- The most promising way to distinguish DM from neutrinos, in particular from solar neutrinos, is the to measure the direction of the nuclear recoil [41, 42]: the Solar system motion through the Galaxy produces a forward-backward asymmetry in the DM signal, that is to some degree reflected in a preferred nuclear recoil direction. An experiment able to measure the direction of the recoils could get rid of other backgrounds, in particular from neutrinos coming from the Sun. The task is not easy because the track of a recoiling nucleus is very short. Even in a low-pressure gaseous detector, a $\sim 1 \mathrm{keV}$ nuclear recoil is about $0.1 \mathrm{~mm}$ length and suffers a quite large diffusion during the drift to the readout plane [43]. Between the pioneers experiments implementing directionality there were the low-pressure gas TPC DRIFT experiment, in UK [44], MIMAC [45] (an electron-drift TPC read by micromegas technology), and NEWAGE [46], another electro-drift TPC at Kamioka. Subsequently other experiments as DMTPC [47] appeared. Currently these experiments have joined and included other new technology proposals and now form a large collaboration called CYGNUS. Another important project searching for directionality is NEWs, a spherical TPC [48]. But there are also many other ideas apart from the gaseous TPCs, as can be the use of anisotropic scintillators [49], emulsions [50], columnar recombination in Xe/Ar [51], among others.

\section{Dark Matter annual modulation}

The rate of interaction of the WIMPs in the detector depends on their relative velocity with respect to the Earth. As a consequence of the Earth rotation around the Sun, this velocity varies along the year, being largest around 2 June and smallest in December [52, 53] (see Fig. 3 panels a and b). Consequently, the amount of particles able to produce nuclear recoils above the energy threshold of the detector is also largest in June. The Earth moves around the Sun following an almost circular orbit with $v_{\text {orb }}=29.8 \mathrm{~km} / \mathrm{s}$ in a plane that is tilted approximately 60 deg with respect to the galactic plane (see panel c) of Fig. 3). A good approximation for the module of the Earth's velocity respect to the rest frame of the dark matter $v_{\oplus}$ is [54]

$$
v_{\oplus} \approx v_{\odot}+b v_{o r b} \cos \omega\left(t-t_{0}\right),
$$

where $v_{\odot}$ is the velocity of the Sun relative to that frame, $\omega=2 \pi / \mathrm{yr}, b \sim 0.5$ and $t_{0}=0.4147$ is the fraction of the year at which $v_{\oplus}$ is maximized (June the $2^{\text {nd }}$ ).

Introducing this expression in the differential scattering rate and expanding in Fourier series to first order, the annually modulating recoil rate can be approximated by a constant term $S_{0}$ plus a modulated term $S_{m}$ with one year period and maximum around June the $2^{\text {nd }}$ :

$$
\frac{d R}{d E}(E, t) \approx S_{0}(E)+S_{m}(E) \cos \omega\left(t-t_{0}\right) .
$$

This approximation is valid for the simplest assumptions about the dark matter distribution in the halo. The modulated amplitude can then be calculated as

$$
S_{m}(E)=\frac{1}{2}\left(\frac{d R}{d E}\left(E, t_{0}\right)-\frac{d R}{d E}\left(E, t_{\min }\right)\right),
$$

where $t_{\min }=t_{0}+0.5 \mathrm{yr}$. It can be positive or negative depending on the WIMP mass and the recoil energy, and in general is a small fraction of $S_{0}(5-7 \%)$ (see panels d) and e) in Fig. 3). The annual 
a) SUMMER

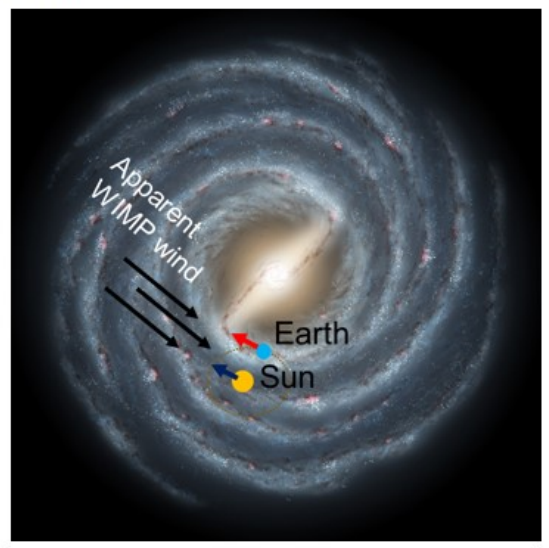

b) WINTER

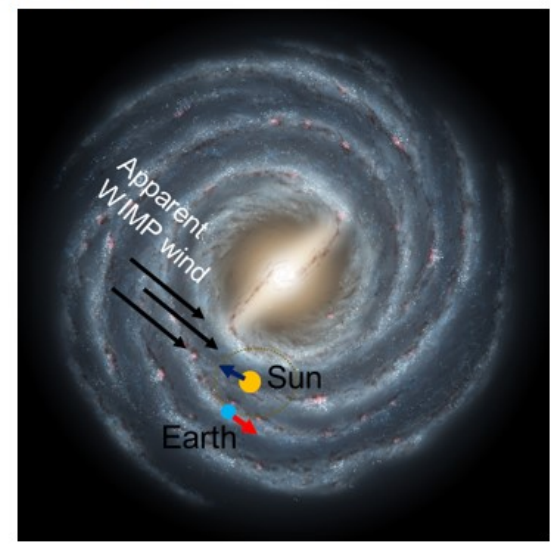

c)
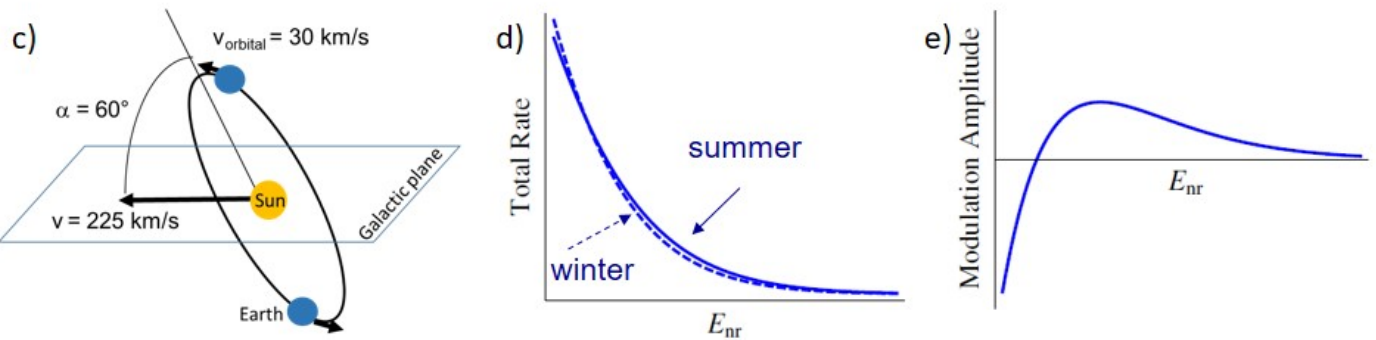

Figure 3: Upper panels: simplified representation of the Sun and Earth movements with respect to the galactic halo in summer (left) and winter (right), which originate the annual modulation in the dark matter detection rate. The modules of the velocities and inclination of the terrestrial orbit with respect to the galactic plane are shown in panel c). Panel d) shows the total detection rate as a function of the recoil energy in a generic detector in summer (solid line) and winter (dashed line). Finally panel e) represents a generic modulation amplitude as a function of energy. At a certain energy (depending on the target) there is a phase inversion.

modulation effect is week in amplitude, but have very peculiar characteristics (e.g. period one year, phase around $2^{\text {nd }}$ of June, noticeable only at very low energy) that make it hard to mimic by any background.

\subsection{The DAMA/LIBRA signal}

For more than twenty years, the DAMA experiment [55] has been claiming the observation of an annual modulation in their detection rate compatible with all the requirements expected for a dark matter signal. The experiment consists of $\mathrm{NaI}(\mathrm{Tl})$ scintillators forming a matrix and is located in the Gran Sasso Underground Laboratory (LNGS). The light produced by a particle interaction in one the crystals is read by two photomultipliers coupled to each scintillator through $10 \mathrm{~cm}$ long light guides. The first stage of the experiment (DAMA) consisted of $\sim 100 \mathrm{~kg}$ of $\mathrm{NaI}(\mathrm{Tl})$ forming a $3 \times 3$ matrix. After 7 years of operation [56] they reported a very clear modulation signal in the 2 to $6 \mathrm{keV}$ region, with $6.3 \sigma$ C.L. In the second step of the experiment, called DAMA/LIBRA, they increased the mass of the detector up to $250 \mathrm{~kg}$, forming a $5 \times 5$ matrix. The previous measured 
modulation was confirmed with the new set-up, reaching 9.3 $\sigma$ C.L. after 7 more years of operation $[57,58]$. Then they performed an upgrade in the detector (DAMA/LIBRA-phase2) changing the photomultipliers for others with higher quantum efficiency [59]. As a result, the energy threshold of the experiment improved from $2 \mathrm{keV}$ to $1 \mathrm{keV}$. DAMA/LIBRA collaboration has recently published the results for 7 additional cycles with phase-2. The modulation is still present with the same characteristics [30] and the signal has reached a $12 \sigma$ C.L.

This impressive result however is in clear contradiction with the negative results of other experiments. The region in the WIMP parameter space singled out by the DAMA/LIBRA positive result, when interpreted in terms of a WIMP signal, is completely excluded by other experiments (see Fig. 1). Some experiments have also reported a search for annual modulation in their data, switching off the electron recoil discrimination in order to decrease their energy threshold and better compare with the DAMA signal (note that the DAMA detector has no electron vs nuclear recoil discrimination; this kind of analysis is possible with scintillators at high energy, based on the different pulse shape produced by the different interacting particles, but at low energy the differences are tiny and do not allow an event-by-event rejection). Those analysis are also in strong tension with the modulation observed by DAMA [60, 61, 62, 63].

Nevertheless, all the above mentioned experiments register the signal in a target different from the DAMA/LIBRA one. As we have revised in section 2, the WIMP expected rate depend upon the target material, so the comparison of the DAMA/LIBRA signal with all the other negative results depends on assumptions about the dark matter composition, interaction and distribution. To ascertain whether the DAMA/LIBRA positive signal is due to WIMPs, an independent confirmation is needed from other experiments using the same target as DAMA and the same technique.

\subsection{Testing the DAMA signal}

There are several experiment around the world pursuing this goal, but only two of them are in data taking: the COSINE-100 experiment, in South Korea, and the ANAIS-112 experiment in Spain. Other experiments looking for the same objective but still in prototype phases are SABRE and COSINUS.

SABRE [64] projects the construction of two twin ultra-pure $\mathrm{NaI}(\mathrm{Tl})$ detectors, one in the LNGS, and the other one in the South hemisphere (Stawell Underground Physics Laboratory (SUPL), in Australia). In this way this experiment will be able to test the potential modulation originated by seasonal-related backgrounds. COSINUS [65] on the other hand propose to use a different technique: NaI bolometers, which with a relative efficiency factor for nuclear recoils against electron recoils very close to 1 would allow to exploit very low energy thresholds and explore the DAMA/LIBRA region with reduced exposure.

COSINE-100 [66] is located at the Yangyang Underground Laboratory in South Korea and started the data-taking in October 2016. The experiment consist of eight $\mathrm{NaI}(\mathrm{Tl})$ crystals of different masses and dimensions, arranged in a $4 \times 2$ array, with a total mass of $106 \mathrm{~kg}$. The detector is immersed in a liquid scintillator tank for the identification and subsequent reduction of radioactive backgrounds. The tank is surrounded by copper, lead and plastic scintillators acting as muon veto. An analysis of the first 59.5 live days, together with a precise background model of the detector [67], allowed the COSINE collaboration to exclude the $3 \sigma$ DAMA/LIBRA region at $90 \%$ C.L. in a model dependent way [68]. They have also reported an annual modulation study using 
$61.3 \mathrm{~kg}$ and 1.7 years of data [69], being the results not conclusive (compatible with both DAMA signal and absence of modulation).

ANAIS-112 experiment [70], at the Canfranc Underground Laboratory (LSC), uses nine NaI(Tl) modules produced by Alpha Spectra Inc. (AS) in Colorado (US). These modules, made of ultrapure NaI powder, have been the result of a long effort, carried out together with AS, to achieve the best detector performance and background level $[71,72,73,74,75,76]$. Each crystal is cylindrical (4.75" diameter and 11.75" length), with a mass of $12.5 \mathrm{~kg}$. The encapsulation has a Mylar window allowing low energy calibration using external gamma sources. The ANAIS-112 shielding consists of $10 \mathrm{~cm}$ of archaeological lead, $20 \mathrm{~cm}$ of low activity lead, an anti-radon box (continuously flushed with radon-free nitrogen gas), an active muon veto system made up of 16 plastic scintillators designed to cover top and sides of the whole ANAIS set-up and $40 \mathrm{~cm}$ of neutron moderator (a combination of water tanks and polyethylene blocks). Thanks to the very high light yield of the crystals (around 15 photoelectrons per keV), the analysis threshold is established at 1 keVee. ANAIS-112 started taking data on August 2017. The experiment performances can be found in [77]. ANAIS collaboration has recently reported their first model-independent annual modulation results [78], corresponding to $112.5 \mathrm{~kg}$ of target mass and 1.5 years of data, amounting to $157.55 \mathrm{~kg} \times$ yr. ANAIS-112 data are consistent with the null hypothesis. The best fits for the modulation hypothesis are consistent with the absence of modulation, resulting in modulation amplitudes of $-0.0044 \pm 0.0058 \mathrm{cpd} / \mathrm{kg} / \mathrm{keV}$ and $-0.0015 \pm 0.0063 \mathrm{cpd} / \mathrm{kg} / \mathrm{keV}$ for [2-6] and [1-6] keV energy ranges, respectively. The best fits in both energy regions are incompatible at $2.5 \sigma(1.9 \sigma)$ with the DAMA/LIBRA signal. The current sensitivity to the DAMA/LIBRA result, determined by the standard deviation of the modulation amplitude distribution, is $1.75 \sigma(1.66 \sigma)$ respectively, in agreement with the estimated sensitivity for the accumulated exposure [79], supporting the projection of reaching a $3 \sigma$ sensitivity to the DAMA/LIBRA result in 5 years of data taking.

\section{Outlook}

The evidence of dark matter is well established due to the gravitational effects it produces, but its nature is still unknown to us. Intense experimental programs are being carried out all over the world to direct detect dark matter particles with a detector on the Earth, with negative results so far despite the impressive sensitivities that have been reached. Next generation experiments will reach the neutrino floor in the next decade, facing a new background, so new strategies are being pursued to go beyond. Innovative experimental ideas are being developed to explore also other parametric regions not at reach with conventional detectors. On the other hand, the only positive signal up to date, provided by the DAMA/LIBRA experiment, is still in strong tension with the negative results of other experiments using other targets. However it could be very soon confirmed or refuted in a model-independent way, as other experiments (ANAIS and COSINE) using the same target have already started their data taking.

\section{References}

[1] G. Bertone and D. Hooper, History of dark matter, Rev. Mod. Phys. 90 (2018) 045002 [arXiv:1605.04909]. 
[2] PLANCK collaboration, Planck intermediate results - XLV. Radio spectra of northern extragalactic radio sources, Astron. Astrophys. 596 (2016) A106 [1606.05120].

[3] B. D. Fields, The primordial lithium problem, Ann. Rev. Nucl. Part. Sci. 61 (2011) 47 [1203. 3551 ].

[4] M. Boudaud and M. Cirelli, Voyager $1 e^{ \pm}$Further Constrain Primordial Black Holes as Dark Matter, Phys. Rev. Lett. 122 (2019) 041104 [1807.03075].

[5] G. Jungman, M. Kamionkowski and K. Griest, Supersymmetric dark matter, Phys. Rept. 267 (1996) $195[$ hep-ph/9506380].

[6] D. Hooper and S. Profumo, Dark matter and collider phenomenology of universal extra dimensions, Phys. Rept. 453 (2007) 29 [hep-ph/ 0701197$].$

[7] R. D. Peccei and H. R. Quinn, CP conservation in the presence of pseudoparticles, Phys. Rev. Lett. 38 (1977) 1440.

[8] A. Ringwald, Exploring the Role of Axions and Other WISPs in the Dark Universe, Phys. Dark Univ. 1 (2012) 116 [1210.5081].

[9] M. Battaglieri et al., US Cosmic Visions: New Ideas in Dark Matter 2017: Community Report, in U.S. Cosmic Visions: New Ideas in Dark Matter College Park, MD, USA, March 23-25, 2017, 2017, 1707.04591 , http://1ss.fnal.gov/archive/2017/conf/fermilab-conf-17-282-ae-ppd-t.pdf.

[10] J. I. Read, The Local Dark Matter Density, J. Phys. G41 (2014) 063101 [1404.1938].

[11] P. Klos, J. Menéndez, D. Gazit and A. Schwenk, Large-scale nuclear structure calculations for spin-dependent wimp scattering with chiral effective field theory currents, Phys. Rev. D 88 (2013) 083516.

[12] S. Kang, S. Scopel, G. Tomar and J.-H. Yoon, Present and projected sensitivities of Dark Matter direct detection experiments to effective WIMP-nucleus couplings, Astropart. Phys. 109 (2019) 50 [1805.06113].

[13] D. Z. Freedman, Coherent Neutrino Nucleus Scattering as a Probe of the Weak Neutral Current, Phys. Rev. D9 (1974) 1389.

[14] COHERENT collaboration, Observation of Coherent Elastic Neutrino-Nucleus Scattering, Science 357 (2017) 1123 [1708.01294].

[15] L. E. Strigari, Neutrino Coherent Scattering Rates at Direct Dark Matter Detectors, New J. Phys. 11 (2009) 105011 [0903.3630].

[16] J. Billard, L. Strigari and E. Figueroa-Feliciano, Implication of neutrino backgrounds on the reach of next generation dark matter direct detection experiments, Phys. Rev. D89 (2014) 023524 [1307.5458].

[17] L. Baudis, Dark matter detection, J. Phys. G43 (2016) 044001.

[18] T. Marrodán Undagoitia and L. Rauch, Dark matter direct-detection experiments, J. Phys. G43 (2016) 013001 [1509.08767].

[19] M. Schumann, Direct Detection of WIMP Dark Matter: Concepts and Status, 1903.03026.

[20] XENON collaboration, Dark Matter Search Results from a One Ton-Year Exposure of XENONIT, Phys. Rev. Lett. 121 (2018) 111302 [1805.12562].

[21] LUX collaboration, Results from a search for dark matter in the complete LUX exposure, Phys. Rev. Lett. 118 (2017) 021303 [1608. 07648 ]. 
[22] PANDAX-II collaboration, Dark Matter Results From 54-Ton-Day Exposure of PandaX-II Experiment, Phys. Rev. Lett. 119 (2017) 181302 [1708.06917].

[23] DEAP collaboration, Search for dark matter with a 231-day exposure of liquid argon using DEAP-3600 at SNOLAB, 1902.04048.

[24] DARKSIDE collaboration, Constraints on Sub-GeV Dark-Matter-Electron Scattering from the DarkSide-50 Experiment, Phys. Rev. Lett. 121 (2018) 111303 [1802.06998].

[25] DARKSIDE collaboration, DarkSide-50 532-day Dark Matter Search with Low-Radioactivity Argon, Phys. Rev. D98 (2018) 102006 [1802 . 07198].

[26] SUPERCDMS collaboration, Results from the Super Cryogenic Dark Matter Search Experiment at Soudan, Phys. Rev. Lett. 120 (2018) 061802 [1708.08869].

[27] SUPERCDMS collaboration, New Results from the Search for Low-Mass Weakly Interacting Massive Particles with the CDMS Low Ionization Threshold Experiment, Phys. Rev. Lett. 116 (2016) 071301 [1509.02448].

[28] CRESST collaboration, Results on light dark matter particles with a low-threshold CRESST-II detector, Eur. Phys. J. C76 (2016) 25 [1509.01515].

[29] C. Savage, G. Gelmini, P. Gondolo and K. Freese, Compatibility of DAMA/LIBRA dark matter detection with other searches, JCAP 0904 (2009) 010 [0 808 . 3607 ].

[30] R. Bernabei et al., First Model Independent Results from DAMA/LIBRA-Phase2, Universe 4 (2018) 116 [arXiv:1805.10486].

[31] XMASS collaboration, A direct dark matter search in XMASS-I, Phys. Lett. B789 (2019) 45 [1804.02180].

[32] D. C. Malling et al., After LUX: The LZ Program, 1110.0103.

[33] DARWIN collaboration, DARWIN: towards the ultimate dark matter detector, JCAP 1611 (2016) $017[1606.07001]$.

[34] DARKSIDE collaboration, Status and Perspective of the DarkSide Experiment at LNGS, Nuovo Cim. C40 (2017) 164.

[35] PTOLEMY collaboration, PTOLEMY: A Proposal for Thermal Relic Detection of Massive Neutrinos and Directional Detection of MeV Dark Matter, 1808.01892.

[36] E. Behnke et al., Final Results of the PICASSO Dark Matter Search Experiment, Astropart. Phys. 90 (2017) 85 [1611.01499].

[37] PICO collaboration, Improved dark matter search results from PICO-2L Run 2, Phys. Rev. D (2016) [1601.03729].

[38] LUX collaboration, Limits on spin-dependent WIMP-nucleon cross section obtained from the complete LUX exposure, Phys. Rev. Lett. 118 (2017) 251302 [1705 . 03380 ].

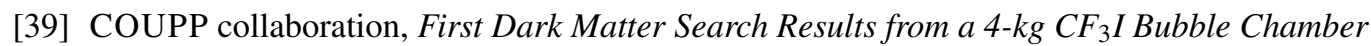
Operated in a Deep Underground Site, Phys. Rev. D86 (2012) 052001 [1204 . 3094 ].

[40] F. Ruppin, J. Billard, E. Figueroa-Feliciano and L. Strigari, Complementarity of dark matter detectors in light of the neutrino background, Phys. Rev. D90 (2014) 083510 [1408.3581].

[41] D. N. Spergel, The Motion of the Earth and the Detection of Wimps, Phys. Rev. D37 (1988) 1353. 
[42] C. J. Copi, J. Heo and L. M. Krauss, Directional sensitivity, WIMP detection, and the galactic halo, Phys. Lett. B461 (1999) 43 [hep-ph/9904499].

[43] F. Mayet et al., A review of the discovery reach of directional Dark Matter detection, Phys. Rept. 627 (2016) 1 [1602.03781].

[44] DRIFT, UK DARK MATTER collaboration, DRIFT: A directionally sensitive dark matter detector, Nucl. Instrum. Meth. A513 (2003) 226.

[45] F. Mayet, O. Guillaudin, C. Grignon, C. Koumeir, D. Santos, P. Colas et al., Micromegas micro-TPC for direct Dark Matter search with MIMAC, J. Phys. Conf. Ser. 179 (2009) 012011 [0 905.4654 ].

[46] A. Takeda, T. Tanimori, H. Kubo, K. Miuchi, T. Nagayoshi, Y. Okada et al., NEWAGE project: Dark matter detection with an advanced gaseous tracking device, in Origin of matter and evolution of galaxies. Proceedings, International Symposium, OMEG'03, Wako, Japan, November 17-19, 2003, pp. 537-540, 2003.

[47] S. Ahlen et al., First Dark Matter Search Results from a Surface Run of the 10-L DMTPC Directional Dark Matter Detector, Phys. Lett. B695 (2011) 124 [1 006 .2928].

[48] NEWS-G collaboration, First results from the NEWS-G direct dark matter search experiment at the LSM, Astropart. Phys. 97 (2018) 54 [1706.04934].

[49] F. Cappella et al., On the potentiality of the $\mathrm{ZnWO}_{4}$ anisotropic detectors to measure the directionality of Dark Matter, Eur. Phys. J. C73 (2013) 2276.

[50] NEWSDM collaboration, Discovery potential for directional Dark Matter detection with nuclear emulsions, Eur. Phys. J. C78 (2018) 578 [1705.00613].

[51] M. Cadeddu et al., Directional dark matter detection sensitivity of a two-phase liquid argon detector, JCAP 1901 (2019) 014 [1704.03741].

[52] A. K. Drukier, K. Freese and D. N. Spergel, Detecting Cold Dark Matter Candidates, Phys. Rev. D33 (1986) 3495.

[53] K. Freese, J. A. Frieman and A. Gould, Signal Modulation in Cold Dark Matter Detection, Phys. Rev. D37 (1988) 3388.

[54] K. Freese, M. Lisanti and C. Savage, Colloquium: Annual modulation of dark matter, Rev. Mod. Phys. 85 (2013) 1561 [arXiv:1209.3339].

[55] R. Bernabei et al., The DAMA/LIBRA apparatus, Nuclear Instruments and Methods in Physics Research A 592 (2008) 297 [arXiv: 0804 .2738].

[56] R. Bernabei et al., Dark matter search, Riv. Nuovo Cim. 26N1 (2003) 1 [astro-ph/0307403].

[57] DAMA collaboration, First results from DAMA/LIBRA and the combined results with DAMA/NaI, Eur. Phys. J. C56 (2008) 333 [arXiv:0804.2741].

[58] R. Bernabei et al., Final model independent result of DAMA/LIBRA-phase1, Eur. Phys. J. C73 (2013) 2648 [arXiv:1308.5109].

[59] R. Bernabei et al., Performances of the new high quantum efficiency PMTs in DAMA/LIBRA, JINST 7 (2012) P03009.

[60] LUX collaboration, Search for annual and diurnal rate modulations in the LUX experiment, Phys. Rev. D98 (2018) 062005 [arXiv: 1807.07113$].$ 
[61] XMASS collaboration, Direct dark matter search by annual modulation with 2.7 years of XMASS-I data, Phys. Rev. D97 (2018) 102006 [arXiv: 1801.10096 ].

[62] XENON collaboration, Search for Electronic Recoil Event Rate Modulation with 4 Years of XENON100 Data, Phys. Rev. Lett. 118 (2017) 101101 [arXiv: 1701.00769 ].

[63] CDMS-II collaboration, Search for annual modulation in low-energy CDMS-II data, 1203.1309.

[64] SABRE collaboration, SABRE: Dark matter annual modulation detection in the northern and southern hemispheres, Nucl. Instrum. Meth. A845 (2017) 418.

[65] G. Angloher et al., The cosinus project: perspectives of a nai scintillating calorimeter for dark matter search, The European Physical Journal C 76 (2016) 441.

[66] G. Adhikari et al., Initial Performance of the COSINE-100 Experiment, Eur. Phys. J. C78 (2018) 107 [arXiv:1710.05299].

[67] COSINE-100 collaboration, Background model for the NaI(Tl) crystals in COSINE-100, Eur. Phys. J. C78 (2018) 490 [1804.05167].

[68] G. Adhikari et al., An experiment to search for dark-matter interactions using sodium iodide detectors, Nature 564 (2018) 83.

[69] COSINE-100 collaboration, Search for a dark matter-induced annual modulation signal in NaI(Tl) with the COSINE-100 experiment, 1903.10098.

[70] J. Amare et al., The ANAIS-112 experiment at the Canfranc Underground Laboratory, in 15th International Conference on Topics in Astroparticle and Underground Physics (TAUP 2017) Sudbury, Ontario, Canada, July 24-28, 2017, 2017, 1710.03837.

[71] C. Cuesta et al., Analysis of the ${ }^{40} \mathrm{~K}$ contamination in NaI(Tl) crystals from different providers in the frame of the ANAIS project, Int. J. Mod. Phys. A29 (2014) 1443010 [1403. 3580 ].

[72] J. Amaré et al., Cosmogenic radionuclide production in NaI(Tl) crystals, JCAP 1502 (2015) 046 [1411.0106].

[73] J. Amare et al., Background model of NaI(Tl) detectors for the ANAIS Dark Matter Project, in Proceedings, 11th Patras Workshop on Axions, WIMPs and WISPs (Axion-WIMP 2015): Zaragoza, Spain, June 22-26, 2015, pp. 232-235, 2015, 1508 .07907, DOI.

[74] J. Amare et al., Assessment of backgrounds of the ANAIS experiment for dark matter direct detection, Eur. Phys. J. C76 (2016) 429 [arXiv: 1604 . 05587].

[75] J. Amare et al., Cosmogenic production of tritium in dark matter detectors, Astropart. Phys. 97 (2018) $96[1706.05818]$.

[76] J. Amaré et al., Analysis of backgrounds for the ANAIS-112 dark matter experiment, submitted to Eur. Phys. J. C (2018) [arXiv:1812.01377].

[77] J. Amaré et al., Performance of ANAIS-112 experiment after the first year of data taking, Eur. Phys. J. C79 (2019) 228 [1812.01472].

[78] J. Amaré et al., First results on dark matter annual modulation from ANAIS-112 experiment, 1903.03973.

[79] I. Coarasa et al., ANAIS-112 sensitivity in the search for dark matter annual modulation, Eur. Phys. J. C79 (2019) 233 [1812.02000]. 